\title{
Lo que se dice y lo que se hace: una perspectiva rortiana sobre la justificación
}

\author{
JUSTINA DÍAZ LEGASPE \\ Departamento de Filosofía \\ Universidad Nacional de La Plata \\ jus@netverk.com.ar
}

\begin{abstract}
Resumen: En su trabajo acerca del debate Putnam-Rorty, Luis Robledo supone una concepción reduccionista de la justificación en Rorty, y defiende contra ella la independencia entre justificación y consenso, a través del argumento de la posibilidad de un consenso sin justificación. A su juicio, el no sostener una independencia tal conduciría a problemas desfavorables para el pragmatismo. En este trabajo buscaré presentar una interpretación alternativa y no reduccionista de la justificación rortiana, que aún sin reconocer una total independencia entre ambas instancias, no sólo evita estos problemas, sino que además muestra que es poco pragmático el ejemplo proporcionado por Robledo en su argumentación.
\end{abstract}

Palabras clave: justificación, consenso, pragmatismo, práctica

Luis Robledo ${ }^{1}$ retoma algunas ideas del debate Putnam-Rorty ${ }^{2}$ a fin de mostrar que rechazar la posibilidad de error comunitario en la justificación conduciría a consecuencias contraproducentes para la adopción de una postura pragmatista. En este trabajo discutiré este punto, presentando una interpretación alternativa del concepto de justificación rortiano que permitiría seguir sosteniendo la imposibilidad del error comunitario, evitando al mismo tiempo las consecuencias indeseables para una teoría pragmatista de dicha práctica.

Robledo considera que los siguientes puntos son fundamentales para cualquier teoría pragmatista de la justificación:

${ }^{1}$ En su artículo “¿Puede realmente una mayoría estar equivocada?”, pp. 143-149, en este número.

${ }^{2}$ Si bien las ideas tanto de Putnam como de Rorty acerca de la justificación pueden encontrarse a lo largo de sus obras, el lector podrá hallar los puntos más relevantes de esta polémica en los siguientes artículos: H. Putnam, "Richard Rorty on Reality and Justification"; R. Rorty, "Response to Hilary Putnam" (ambos en R. Brandom (comp.), Rorty and His Critics, Blackwell, 2000, pp. 81-86 y 87-90, respectivamente), y en R. Rorty, "Putnam and the Relativist Menace", The Journal of Philosophy, vol. 90, no. 9, 1993, pp. 443-461. 
(1) El carácter contextual de la justificación.

(2) La afirmación rortiana de que normas y estándares de justificación deben ser buscados en las prácticas lingüísticas de la comunidad.

(3) La independencia entre consenso y justificación, puesto que la reducción de la segunda al primero lleva inevitablemente a considerar la imposibilidad del error en la práctica de justificación de la comunidad.

Cabe hacer aquí algunas aclaraciones respecto de estos puntos: Robledo parte del supuesto de que Rorty reduce la justificación al consenso, identificando así la práctica de justificación efectiva de la comunidad con la práctica correcta de justificación. Tal como lo resalta Kalpokas, ${ }^{3}$ al definir "objetividad de la justificación" como "observable desde una perspectiva sociológica", Rorty estaría entonces admitiendo que aquello que una comunidad hace cuando alcanza un consenso al justificar coincide con la justificación correctamente realizada, idea que conduciría a un infalibilismo respecto de la justificación, a una negativa a admitir que una comunidad puede estar equivocada a pesar de haber logrado un consenso sobre la justificación de cierta oración. Por ello, rechaza la reducción rortiana de la justificación al consenso y reafirma la tesis putnamiana de la independencia entre ambas, presentando para ello el ejemplo de la comunidad $C$.

Por otro lado, el hecho de que las normas de justificación sean explícitamente enunciadas y admitidas como tales por la comunidad permite a Robledo mantener la contextualidad de la justificación (puesto que no es un intérprete externo quien determina cuáles son estas normas, sino los mismos miembros de la comunidad), y le permite además realizar una interesante distinción entre dos tipos diferentes de prácticas lingüísticas, a fin de sostener consistentemente la suposición rortiana de que normas y estándares de justificación deben buscarse en las prácticas lingüísticas de cada comunidad. Robledo parece diferenciar implícitamente dos tipos de prácticas: aquello que la comunidad hace cuando justifica, esto es, un intercambio de razones conducente en última instancia al consenso global acerca de $p$, y aquello que la comunidad dice que hace cuando justifica, esto es, la enunciación de los tests al preguntársele a cualquier miembro de $C$ por sus normas de justificación. Rorty parecería afirmar que es el primer tipo de práctica en el que deben buscarse las normas de justificación; Robledo, por su parte, parece considerar que esto implicaría volver a la identificación de justificación y consenso, con lo cual, para seguir sosteniendo consistentemente la relación entre normas y prácticas lingüísticas y el contextualismo de la justificación, sostiene que es el segundo tipo de

${ }^{3}$ En D. Kalpokas, “El debate Putnam-Rorty sobre la naturaleza de la justificación”, pp. 119126 , en este mismo número. 
práctica la que proporcionarían al intérprete externo las normas de justificación propias de una comunidad.

La presentación de Robledo del caso de la comunidad $C$ resulta útil para sus propósitos sobre el supuesto de que Rorty se vería obligado, tras reducir la justificación al consenso, a afirmar que la comunidad justifica $p$ a pesar de que $p$ no satisface una norma epistémica, y a pesar de que por hipótesis se sostiene que cualquier miembro de la comunidad, al ser advertido de dicha omisión, afirmaría que $p$ no está correctamente justificado. De este modo Robledo muestra la independencia de la justificación (a través de los tests) respecto del consenso al que llega la comunidad. La negativa de Rorty a reconocer esta independencia y el consecuente rechazo de la posibilidad de error comunitario conduciría a las siguientes dificultades:

a) De negarse la posibilidad de error comunitario, debería admitirse que dada una comunidad que en un tiempo 1 respetara el valor de toda vida humana o de las minorías, si ésta pasara en un tiempo 2 a justificar un régimen nazi, no podría admitirse que la comunidad se equivoca (ni siquiera desde la posición de un intérprete externo que buscara las normas de justificación en las prácticas lingüísticas que llevaron a este consenso). Esto ataca la idea misma de reforma intracomunitaria de la justificación.

b) Robledo ve como una contradicción práctica el que, a fin de negar que la comunidad se equivoca, la salida más fácil sea la de afirmar que la comunidad se equivoca, no ya en las prácticas lingüísticas que conducen al consenso, sino en la práctica lingüística de afirmar cuáles son las normas de justificación que se siguen al justificar.

c) El rechazo a la posibilidad del error comunitario conduciría a la violación del principio sostenido en (1), puesto que es el intérprete externo quien afirma que las normas de la comunidad están insertas en lo que la comunidad hace al justificar, aun cuando los miembros de la comunidad afirmen que sus normas son tanto aquellas que se tuvieron en cuenta en la justificación como aquellas que no. Esto constituye, para Robledo, una negación de la autoridad epistémica de la comunidad sobre sus prácticas de justificación.

Sin embargo, considero que una reconstrucción alternativa del concepto rortiano de justificación mantiene los puntos (1) y (2) sostenidos antes, y disuelve los problemas recién mencionados, y volviendo innecesario seguir manteniendo el punto (3), cuya función es la de evitar el infalibilismo en la justificación, que conduciría a dichos problemas. Esta interpretación no sólo no es contraproducente para el pragmatismo, sino que posee aún un carácter más pragmatista que el concepto de justificación presentado por Robledo. 
Tanto Robledo como Putnam arriban a muchos problemas por causa de una mala interpretación del concepto de objetividad de la justificación presentado por Rorty. Este filósofo presenta una definición reformada de "objetividad", según la cual "objetivo" equivale a ser detectable a través de las prácticas de la comunidad, lo cual le permite admitir que la justificación es una "cuestión sociológica"4 objetiva, al menos en este sentido del término. El error de Putnam y de Robledo reside en confundir la estrecha relación que Rorty propone entre justificación y consenso observable externamente, con una reducción de la justificación al consenso. De la afirmación rortiana de que la justificación es observable no se sigue necesariamente que no haya más en la justificación que el mero consenso global de la comunidad. Para Rorty, la justificación consiste en el intercambio de razones entre los miembros de una comunidad, en un juego de dar y recibir razones que podría llevar en última instancia a un consenso total de la comunidad, siempre y cuando no hubiera más razones que alegar, ni nadie que pudiera proporcionar nuevas razones no tenidas en cuenta en el proceso de justificación. Así, el consenso total es sólo el rostro empírico de la justificación, a través del cual podemos afirmar que dicho proceso ha sido completado. La acusación de reduccionismo que se le hace a Rorty no tiene en cuenta que el consenso puede darse por varios motivos subyacentes, pero que sólo debe considerarse como signo observable de la justificación cuando es producido por este juego de intercambiar razones. Dichas razones se apoyan en las normas de justificación pertenecientes a las prácticas normativas de la comunidad, de modo tal que no cualquier razón será considerada válida en el contexto de cada juego de justificar. Esto permite a Rorty afirmar la "objetividad" de la justificación (en cuanto que tiene un rostro empírico) sin dejar de lado el aspecto normativo que conduce a dicho consenso y que está inserto en la práctica de justificación de la comunidad.

Por otro lado, volviendo a la distinción realizada antes entre lo que la comunidad hace y lo que dice que hace al justificar, considero que, desde una perspectiva pragmatista de la justificación, el intérprete externo, al buscar las normas de justificación de una comunidad, debe volcarse a lo que la comunidad hace efectivamente, dejando de lado aquellas normas que no son aplicadas en el proceso de justificación. Esto se debe, por un lado, al concepto rortiano de justificación como juego de intercambio de razones, razones que son elegidas por cada miembro sobre la base de las prácticas normativas de su comunidad; esto implica que, por su pertenencia a una determinada comunidad, el sujeto conocería de antemano cuáles razones son válidas y es legítimo esgrimirlas en una determinada discusión y cuáles no. Si esto es así, sólo las razones y las normas que

${ }^{4}$ R. Rorty, "Putnam and the Relativist Menace". 
surgen en el juego de justificación son las que se tienen en cuenta para determinar la justificación de una creencia: aquellas razones que no surgen en la discusión no fueron consideradas relevantes para la justificación de la oración en cuestión, puesto que de otro modo aún habría argumentos que considerar y, por tanto, la justificación no estaría completa, lo cual se traduciría, empíricamente, en la ausencia de un consenso comunitario. En el ejemplo de Robledo debería considerarse, entonces, que si no se tuvo en cuenta una de las normas de justificación, fue porque la comunidad no la consideró relevante para justificar $p$. Éste es precisamente el punto que Robledo rechaza como salida válida al problema en (b). Para mostrar que no hay contradicción práctica es preciso detenerse en un principio pragmatista que Robledo parece haber pasado por alto, formulado por primera vez por Peirce ${ }^{5}$ y popularizado por James, ${ }^{6}$ según el cual algo que no hace diferencia en la práctica no debería hacer diferencia tampoco en filosofía. La aplicación de este principio a la distinción entre lo que la comunidad hace y lo que la comunidad dice que hace cuando justifica nos permite afirmar que aquellas razones que no hacen diferencia en la práctica, esto es, que no llegan a ingresar nunca en el juego de intercambiar razones, no deben ser tomadas en cuenta al realizar un examen filosófico de la justificación. La salida al ejemplo proporcionado por Robledo no necesariamente implica la atribución de un error a la comunidad respecto de cuáles son las normas con las cuales justifican, sino más bien señala que éstas difieren de las normas que realmente se aplican. De este modo, el test 10 es sostenido sólo nominalmente por la comunidad, puesto que a ninguno de los miembros les pareció importante traerlo a colación a la hora de justificar $p$. Y puesto que no hizo ninguna diferencia en la práctica, no debería tenérselo en cuenta como norma genuina de justificación.

Esta misma consideración permite evadir la acusación de violación del contextualismo de la justificación, puesto que no se estaría negando la autoridad epistémica de dicha comunidad; un intérprete sólo debería considerar como normas aquellas que los miembros de la comunidad pusieron en juego en las razones dadas que llevaron al consenso global sobre $p$, puesto que las normas meramente enunciadas no hacen en realidad el juego de la justificación. Más allá de qué normas aleguen usar los miembros de la comunidad, sólo se considerarán como normas efectivas aquellas que se ponen en práctica.

Respecto del último problema señalado por Robledo, es preciso recordar la relación entre el contextualismo de la justificación, y la unión entre los valores e intereses de una comunidad y su modo de justificar. El ejemplo

${ }^{5}$ C.S. Peirce, "The Fixation of Belief" [1877] y "How to Make our Ideas Clear" [1878], en N. Houser y C. Kloesel (comps.), The Essential Peirce, vol. I, Indiana University Press, Bloomington, 1992, pp. 109-123 y 124-141, respectivamente.

${ }^{6}$ W. James, Pragmatism [1907], Dover, Nueva York, 1995, cap. 1. 
de la comunidad nazi busca afirmar que, a pesar del consenso global, la comunidad se equivoca. Si bien éste es uno de los puntos más difíciles de defender desde una posición rortiana, es posible mostrar la imposibilidad de este ejemplo. Al contestar a una crítica similar por parte de Putnam, Rorty alega que, en cualquier caso, existe un criterio contra el cual podemos contrastar nuestras decisiones a la hora de justificar, a fin de evaluar si éstas supusieron una mejora o no respecto de nuestro estado anterior. Este criterio estaría dado por imaginar qué habría decidido la "mejor versión de nosotros mismos". ${ }^{7}$ Dicha comunidad ideal ${ }^{8}$ compartiría con la nuestra las normas de justificación, pero también los intereses y los valores. Ahora bien, con los valores y los intereses sucede lo mismo que con las normas de justificación: desde una perspectiva pragmatista, no podemos considerar como valores a aquellos que no llevan a ninguna diferencia en la práctica, esto es, aquellos que no aplicamos a la hora de actuar. También aquí deben diferenciarse los valores que una comunidad dice que posee, de los valores con que la comunidad se maneja efectivamente en sus acciones. Así, si una comunidad justifica no sólo con base en normas, sino también en valores e intereses, y si éstos son sólo aquellos que se ponen en práctica y no los que meramente se enuncian, entonces está claro que la comunidad que realmente considere valioso el respeto a las minorías o la vida de todos los miembros por igual, no llegaría a consensuar la justificación de un régimen nazi, puesto que, de ser efectivos dichos valores, surgirían en el intercambio de razones en algún momento. Cuando dichos valores no surgen y se permite la instauración de un régimen nazi, se debe simplemente a que dichos valores eran meramente nominales, sin efectos prácticos. De este modo, el ejemplo proporcionado por Robledo se vuelve inconcebible para una comunidad que realmente ponga en práctica los valores que condenan el nazismo. Cuando pensamos en una comunidad con nuestros mismos valores efectivos, no podemos considerar que llegaría a justificar el régimen nazi, al menos no sin abandonar previamente dichos valores, y dejando por tanto de ser una versión de nosotros mismos. Tal vez no sea ésta la solución para el problema que plantea el concepto de justificación rortiano acerca de la reforma intracomunitaria, pero al menos elimina la posibilidad de algunos de los casos extremos señalados por Putnam.

${ }^{7}$ Sobre este punto en particular, véanse los trabajos de F. Penelas, "La justificación como hecho social", y G. Satne, "Una defensa de la justificación etnocéntrica", ambos en las pp. 127134 y pp. 135-142, respectivamente, en este número.

8 Este término está tomado aquí en el sentido de "mejor versión de nosotros mismos", por lo cual no debe interpretarse en un sentido putnamiano. 
III

En conclusión, partiendo de un concepto de justificación rortiano, podemos aun así mantener el rechazo a la posibilidad de error comunitario cuando se logra un consenso, y sin embargo no considerar contraproducentes aquellos puntos que Robledo señala como tales. Al contrario, la aplicación del principio pragmatista al ejemplo de Robledo muestra cómo la separación entre consenso y justificación correcta lleva a la consideración de un reino de valores y normas que no usamos y que sostenemos por sobre nuestras prácticas efectivas sin que causen ningún efecto en ellas. Tales normas o valores no deberían ser considerados como tales desde una perspectiva pragmatista que se propone ajustarse a las prácticas humanas, en lugar de especular cómo deberían ser éstas.

Recibido el 19 de junio de 2002; aceptado el 26 de noviembre de 2002 